\title{
Time-Dependent Coupled-Cluster Calculations of Polarizabilities and Dispersion Energy Coefficients
}

\author{
RICHARD J. WHEATLEY \\ School of Chemistry, The University of Nottingham, Nottingham, United Kingdom, NG7 2RD
}

Received 22 March 2007; Accepted 11 June 2007

DOI 10.1002/jcc.20801

Published online in Wiley InterScience (www.interscience.wiley.com).

\begin{abstract}
Time-dependent coupled cluster theory, with unrestricted electron spins and full treatment of orbital rotation, is used to calculate polarizabilities at imaginary frequencies for $\mathrm{Li}, \mathrm{Ar}, \mathrm{HCl}, \mathrm{CO}, \mathrm{N}_{2}, \mathrm{O}_{2}$, and $\mathrm{H}_{2} \mathrm{O}$, and to obtain dispersion energy coefficients for their pair interactions. Results obtained with augmented quadruple-zeta basis sets agree well with the best literature values of the $C_{6}$ dispersion energy coefficients. Time-dependent coupled cluster with single and double excitations theory will be useful as a benchmark for evaluating more approximate theories.
\end{abstract}

(C) 2007 Wiley Periodicals, Inc. J Comput Chem 00: 000-000, 2007

Key words: coupled cluster; frequency dependent polarizability; dispersion coefficient; oxygen

\section{Introduction}

The dispersion interaction energy $E_{\text {disp }}$ plays a leading role in van der Waals interactions between nonpolar molecules. At sufficiently large intermolecular separations $R$, it can be approximated well by the first few terms of a multipole series,

$$
E_{\text {disp }}=-\sum_{n \geq 6} C_{n} R^{-n}
$$

where $C_{n}$ is a dispersion energy coefficient. Although the multipole series diverges at small separations, the dispersion energy coefficients are still useful for constructing models for the dispersion energy. The terms in eq. (1) can be multiplied by suitable damping functions $f_{n}(R),{ }^{1-4}$ which are 1 for large $R$ and rapidly tend to zero as $R$ decreases, to correct the divergence of the series.

The dispersion energy coefficients $C_{n}$ depend on the orientations of the molecules, but orientation-independent dispersion energy coefficients $C_{\mathrm{disp} ; \lambda_{1}, \lambda_{2} ; \lambda_{3}, \lambda_{4}}$ can be defined, ${ }^{5}$ where $\lambda_{u}$ is a real multipole component $\left(l_{u}, \kappa_{u}\right)$, and $n=l_{1}+l_{2}+l_{3}+l_{4}+2$, and these orientation-independent dispersion energy coefficients can be used to calculate the multipolar dispersion energy at any intermolecular geometry $\left(R_{\mathrm{AB}}, \Omega_{\mathrm{AB}}\right)$

$$
\begin{aligned}
E_{\text {disp,mult, } \mathrm{AB}}\left(R_{\mathrm{AB}}, \Omega_{\mathrm{AB}}\right)= & -\sum_{\lambda_{1}} \sum_{\lambda_{2}} \sum_{\lambda_{3}} \sum_{\lambda_{4}} T_{\lambda_{1}, \lambda_{3}}\left(R_{\mathrm{AB}}, \Omega_{\mathrm{AB}}\right) \\
& \times T_{\lambda_{2}, \lambda_{4}}\left(R_{\mathrm{AB}}, \Omega_{\mathrm{AB}}\right) C_{\mathrm{disp} ; \lambda_{1}, \lambda_{2} ; \lambda_{3}, \lambda_{4}} .
\end{aligned}
$$

The function $T_{\lambda, \lambda^{\prime}}\left(R_{\mathrm{AB}}, \Omega_{\mathrm{AB}}\right)$ is the well-known ${ }^{6}$ interaction between a multipole $Q_{\lambda}$ of molecule A and a multipole $Q_{\lambda^{\prime}}$ of molecule B. It consists of the product of an orientation-dependent function and the inverse power of separation $R_{\mathrm{AB}}^{-l-l^{\prime}-1}$.

An accurate calculation of dispersion energy coefficients is difficult for all but the smallest atoms and molecules. For large $n$, polarization functions with high angular momenta are needed in the basis set, and the calculations are sensitive to the electronic wave function remote from the nuclei, which means that large basis sets with a balance between diffuse and contracted functions are required. The contribution of electron correlation to the dispersion energy coefficients is also important. In this article, the implementation of an orbital-relaxed, spin-unrestricted TD-CCSD (time-dependent coupled cluster with single and double excitations) method for calculating dispersion energy coefficients is described, calculations are performed using different basis sets, and the results are compared with other methods and with calculated and experimental results from the literature. The next section contains a description of the methodology, and the subsequent section presents the results and the conclusions.

Atomic units are used in this article; the atomic unit of length is the Bohr, $a_{0}=5.291772 \times 10^{-11} \mathrm{~m}$, and the atomic unit of energy is the Hartree, $E_{\mathrm{h}}=4.35975 \times 10^{-18} \mathrm{~J}$. The dispersion energy coefficient $C_{n}$ has units of $E_{\mathrm{h}} a_{0}^{n}$.

Correspondence to: R. J. Wheatley; e-mail: richard.wheatley@nottingham. ac.uk

Contract/grant sponsor: Engineering and Physical Sciences Council 


\section{TD-CCSD Theory}

The dispersion energy coefficients for the A-B interaction that appear in eq. (2), can be calculated using an integral of the frequencydependent polarizabilities of $\mathrm{A}$ and $\mathrm{B}$, over imaginary frequencies, using the Casimir-Polder identity:

$$
C_{\mathrm{disp} ; \lambda_{1}, \lambda_{2}, \lambda_{3}, \lambda_{4}}=\frac{1}{2 \pi} \int_{0}^{\infty} \alpha_{\mathrm{A}, \lambda_{1}, \lambda_{2}}(i \omega) \alpha_{\mathrm{B}, \lambda_{3}, \lambda_{4}}(i \omega) d \omega .
$$

The integral is straightforward to evaluate using numerical methods (see the following section), so the problem of calculating dispersion energy coefficients reduces to one of evaluating the imaginaryfrequency-dependent polarizabilities of the interacting molecules. Apart from their use in eq. (3), imaginary-frequency-dependent polarizabilities are not physically useful quantities, ${ }^{5}$ and unlike real-frequency-dependent polarizabilities, they usually exhibit a maximum value at $\omega=0$.

The frequency-dependent polarizability $\alpha_{\mathrm{A}, \lambda, \lambda^{\prime}}(\omega)$ of a molecule $A$ in a nondegenerate ground state is formally defined as a sum over states:

$$
\begin{array}{r}
\alpha_{\mathrm{A}, \lambda, \lambda^{\prime}}(\omega)=2 \sum_{\mathrm{a}>0}\left(E_{\mathrm{A}}^{\mathrm{a}}-E_{\mathrm{A}}^{(0)}\right)\left\langle\psi_{\mathrm{A}}^{(0)}\left|\hat{Q}_{\mathrm{A}, \lambda}\right| \psi_{\mathrm{A}}^{\mathrm{a}}\right)\left\langle\psi_{\mathrm{A}}^{\mathrm{a}}\left|\hat{Q}_{\mathrm{A}, \lambda^{\prime}}\right| \psi_{\mathrm{A}}^{(0)}\right\rangle \\
/\left[\left(E_{\mathrm{A}}^{\mathrm{a}}-E_{\mathrm{A}}^{(0)}\right)^{2}-\omega^{2}\right]
\end{array}
$$

where $\hat{Q}_{\mathrm{A}, \lambda}$ is a multipole operator, $\psi_{\mathrm{A}}^{(0)}$ is the ground-state electronic wave function with energy $E_{\mathrm{A}}^{(0)}, \psi_{\mathrm{A}}^{\mathrm{a}}$ is an excited-state wave function with energy $E_{\mathrm{A}}^{\mathrm{a}}$, and $\omega$ is expressed in energy units. The sum includes all continuum states. This work is concerned only with imaginary frequencies $\omega$, which do not produce singularities in eq. (4).

In practice, the frequency-dependent polarizability can be calculated using perturbation theory. The unperturbed zero-order electronic Hamiltonian $\hat{H}_{\mathrm{A}}^{(0)}$ is modified by adding a time-dependent perturbation $\hat{Q}_{\mathrm{A}}(t)$ :

$$
\hat{H}_{\mathrm{A}}=\hat{H}_{\mathrm{A}}^{(0)}+\mu \hat{Q}_{\mathrm{A}, \lambda}\left(e^{i \omega t}+e^{-i \omega t}\right)+\mu^{\prime} \hat{Q}_{\mathrm{A}, \lambda^{\prime}}\left(e^{i \omega t}+e^{-i \omega t}\right),
$$

and this is inserted into a phase-isolated time-dependent Schrödinger equation ${ }^{7}$

$$
\left(\hat{H}_{\mathrm{A}}-i \frac{d}{d t}\right) \psi_{\mathrm{A}}=E_{\mathrm{A}} \psi_{\mathrm{A}}
$$

where $E_{\mathrm{A}}$ is the quasi-energy.

The wave function and energy are expanded in powers of the perturbation strengths $\mu$ and $\mu^{\prime}$, and in powers of $e^{i \omega t}$, and the standard techniques of perturbation theory can then be used to show that the frequency-dependent polarizability $\alpha_{\mathrm{A}, \lambda, \lambda^{\prime}}(\omega)$ is simply half of the coefficient of the time-independent energy contribution, which is first order in both $\mu$ and $\mu^{\prime}$ (and therefore second order overall).
The energy equation for the TD-CCSD method is based on the Lagrangian formulation ${ }^{8}$ of the CCSD equations. Using a phaseisolated ansatz, the TD-CCSD Lagrangian is

$$
\begin{aligned}
E_{\mathrm{A}}=\left\langle\phi_{\mathrm{A}}^{(0)}\right| & \hat{\Lambda}_{\mathrm{A}} e^{-\hat{T}_{\mathrm{A}}(t)} e^{-\hat{\kappa}_{\mathrm{A}}(t)}\left(\hat{H}_{\mathrm{A}}^{(0)}-i \frac{d}{d t}+\hat{Q}_{A}(t)\right) \\
& \times e^{\hat{\kappa}_{\mathrm{A}}(t)} e^{\hat{T}_{\mathrm{A}}(t)}\left|\phi_{\mathrm{A}}^{(0)}\right\rangle+\left\langle\phi_{\mathrm{A}}^{(0)}\right|\left[\hat{\zeta}_{\mathrm{A}}, e^{-\hat{\kappa}_{\mathrm{A}}(t)}\right. \\
& \left.\times\left(\hat{H}_{\mathrm{A}}^{(0)}-i \frac{d}{d t}+\hat{Q}_{A}(t)\right) e^{\hat{\kappa}_{\mathrm{A}}(t)}\right]\left|\phi_{\mathrm{A}}^{(0)}\right\rangle
\end{aligned}
$$

where $\hat{\Lambda}_{\mathrm{A}}$ is a de-excitation operator and $\hat{\zeta}_{\mathrm{A}}$ is a sum of excitation and de-excitation operators, and their amplitudes are Lagrange multipliers, which are introduced to enforce the usual conditions for the CCSD amplitudes, $T$, and for the TD-CHF orbital rotation operators, $\hat{\kappa}$, respectively. The operator $\hat{T}_{\mathrm{A}}$ is a sum of single and double excitations, multiplied by CCSD amplitudes; $\hat{\Lambda}_{\mathrm{A}}-1$ is a sum of single and double de-excitations, multiplied by $\lambda$ coefficients; $\phi_{\mathrm{A}}^{(0)}$ is the unperturbed Hartree-Fock wave function, and the orbital rotation operators $\hat{\kappa}_{\mathrm{A}}$ are fixed at their TD-CHF values. The Lagrangian $E_{\mathrm{A}}$ is stationary with respect to all Lagrange multipliers, CCSD amplitudes, and orbital rotation coefficients.

Using the stationary properties of this Lagrangian, the required second-order energy can be expressed in a form that contains terms up to first order in amplitudes and orbital rotations, and zero-order Lagrange multipliers:

$$
\begin{aligned}
& E_{\mathrm{A}}^{(2)}=\left\langle\phi_{\mathrm{A}}^{(0)}\left|\hat{\Lambda}_{\mathrm{A}}^{(0)} e^{-\hat{T}_{\mathrm{A}}^{(0)}} \frac{1}{2}\left[\left[\hat{H}_{\mathrm{A}}^{(0)}-i \frac{d}{d t}, \hat{T}_{\mathrm{A}}^{(1)}\right], \hat{T}_{\mathrm{A}}^{(1)}\right] e^{\hat{T}_{\mathrm{A}}^{(0)}}\right| \phi_{\mathrm{A}}^{(0)}\right\rangle \\
& +\left\langle\phi_{\mathrm{A}}^{(0)}\left|\hat{\Lambda}_{\mathrm{A}}^{(0)} e^{-\hat{T}_{\mathrm{A}}^{(0)}}\left[\left[\hat{H}_{\mathrm{A}}^{(0)}-i \frac{d}{d t}, \hat{\kappa}_{\mathrm{A}}^{(1)}\right], \hat{T}_{\mathrm{A}}^{(1)}\right] e^{\hat{T}_{\mathrm{A}}^{(0)}}\right| \phi_{\mathrm{A}}^{(0)}\right\rangle \\
& +\left\langle\phi_{\mathrm{A}}^{(0)}\left|\hat{\Lambda}_{\mathrm{A}}^{(0)} e^{-\hat{T}_{\mathrm{A}}^{(0)}} \frac{1}{2}\left[\left[\hat{H}_{\mathrm{A}}^{(0)}-i \frac{d}{d t}, \hat{\kappa}_{\mathrm{A}}^{(1)}\right], \hat{\kappa}_{\mathrm{A}}^{(1)}\right] e^{\hat{T}_{\mathrm{A}}^{(0)}}\right| \phi_{\mathrm{A}}^{(0)}\right\rangle \\
& +\left\langle\phi_{\mathrm{A}}^{(0)}\left|\hat{\Lambda}_{\mathrm{A}}^{(0)} e^{-\hat{T}_{\mathrm{A}}^{(0)}}\left[\hat{Q}_{A}, \hat{T}_{\mathrm{A}}^{(1)}+\hat{\kappa}_{\mathrm{A}}^{(1)}\right] e^{\hat{T}_{\mathrm{A}}^{(0)}}\right| \phi_{\mathrm{A}}^{(0)}\right\rangle \\
& +\left\langle\phi_{\mathrm{A}}^{(0)}\left|\left[\hat{\zeta}_{\mathrm{A}}^{(0)}, \frac{1}{2}\left[\left[\hat{H}_{\mathrm{A}}^{(0)}-i \frac{d}{d t}, \hat{\kappa}_{\mathrm{A}}^{(1)}\right], \hat{\kappa}_{\mathrm{A}}^{(1)}\right]\right]\right| \phi_{\mathrm{A}}^{(0)}\right\rangle \\
& +\left\langle\phi_{\mathrm{A}}^{(0)}\left|\left[\hat{\zeta}_{\mathrm{A}}^{(0)},\left[\hat{Q}_{A}, \hat{\kappa}_{\mathrm{A}}^{(1)}\right]\right]\right| \phi_{\mathrm{A}}^{(0)}\right\rangle \text {. }
\end{aligned}
$$

The zero-order quantities in this equation are evaluated ${ }^{9}$ by performing a ground-state CCSD calculation, giving $\hat{T}_{\mathrm{A}}^{(0)}$, solving the CCSD lambda equations to obtain $\hat{\Lambda}_{\mathrm{A}}^{(0)}$, and solving the CCSD zeta equations to obtain $\hat{\zeta}_{\mathrm{A}}^{(0)}$. The TD-CHF equations are solved to obtain $\hat{\kappa}_{\mathrm{A}}^{(1)}$.

The calculation of the first-order amplitudes in $\hat{T}_{\mathrm{A}}^{(1)}$ usually dominates the overall computational expense, as they require essentially a separate CCSD calculation for each perturbation. The first-order 
Table 1. Spherically Averaged Dispersion Energy Coefficients (in Atomic Units), Calculated Using the TD-CCSD Method with Unrestricted Electron Spins.

\begin{tabular}{|c|c|c|c|c|c|c|}
\hline Basis set & $\mathrm{DZ}$ & $\mathrm{TZ}$ & QZ & SPTZ & SPQZ & Literature \\
\hline \multicolumn{7}{|l|}{$\mathrm{Li}$} \\
\hline$C_{6}^{0}$ & 1,361 & 1,362 & 1,348 & 1,359 & 1,346 & 1,387 (ref. 14) \\
\hline$C_{8}^{0}$ & 14,290 & 37,830 & 48,270 & 76,590 & 76,820 & \\
\hline \multicolumn{7}{|l|}{$\mathrm{Ar}^{\circ}$} \\
\hline$C_{6}^{0}$ & 57.9 & 63.6 & 64.0 & 64.3 & 65.1 & 64.3 (ref. 15) \\
\hline$C_{8}^{0}$ & 610 & 1,149 & 1,373 & 1,586 & 1,642 & \\
\hline \multicolumn{7}{|l|}{$\mathrm{HCl}$} \\
\hline$C_{6}^{0}$ & 118.4 & 125.0 & 125.3 & 126.8 & 126.7 & 130.4 (ref. 15) \\
\hline$C_{8}^{0}$ & 2,427 & 3,462 & 3,822 & 4,132 & 4,187 & \\
\hline \multicolumn{7}{|l|}{$\mathrm{CO}$} \\
\hline$C_{6}^{0}$ & 78.8 & 79.2 & 78.8 & 79.8 & 79.1 & 81.3 (ref. 16) \\
\hline$C_{8}^{0}$ & 2,719 & 2,975 & 3,032 & 3,106 & 3,090 & \\
\hline \multicolumn{7}{|l|}{$\mathrm{N}_{2}$} \\
\hline$C_{6}^{0}$ & 72.9 & 73.1 & 72.6 & 73.5 & 72.9 & 73.3 (ref. 15) \\
\hline$C_{8}^{0}$ & 2,220 & 2,435 & 2,488 & 2,543 & 2,537 & \\
\hline \multicolumn{7}{|l|}{$\mathrm{O}_{2}$} \\
\hline$C_{6}^{0}$ & 58.0 & 60.7 & 60.9 & 61.1 & 61.2 & 61.6 (ref. 14) \\
\hline$C_{8}^{0}$ & 1,373 & 1,629 & 1,723 & 1,782 & 1,813 & \\
\hline \multicolumn{7}{|l|}{$\mathrm{H}_{2} \mathrm{O}$} \\
\hline$C_{6}^{0}$ & 43.3 & 45.0 & 45.1 & 45.9 & 45.6 & 45.3 (ref. 14) \\
\hline$C_{8}^{0}$ & 832 & 1,052 & 1,109 & 1,162 & 1,174 & \\
\hline
\end{tabular}

Literature $C_{6}^{0}$ values are taken from constrained dipole oscillator strength data.

amplitudes are obtained by making the first-order energy stationary with respect to $\hat{\Lambda}_{\mathrm{A}}^{(0)}$ :

$$
\left\langle\phi_{\mathrm{A}}^{(0)}\left|\hat{X}_{\mathrm{A}} e^{-\hat{T}_{\mathrm{A}}^{(0)}}\left\{\left[\hat{H}_{\mathrm{A}}^{(0)}-i \frac{d}{d t}, \hat{T}_{\mathrm{A}}^{(1)}+\hat{\kappa}_{\mathrm{A}}^{(1)}\right]+\hat{Q}_{A}\right\} e^{\hat{T}_{\mathrm{A}}^{(0)}}\right| \phi_{\mathrm{A}}^{(0)}\right\rangle=0
$$

where $\hat{X}_{\mathrm{A}}$ includes all single and double de-excitations. In this equation, the imaginary part of the first-order operator $\left[\hat{H}_{\mathrm{A}}^{(0)}, \hat{\kappa}_{\mathrm{A}}^{(1)}\right]$ is anti-Hermitian, so some care is needed in ordering the indices of the one-electron and two-electron integrals produced from it. These include integrals with four virtual orbital indices, which would require a large amount of disk storage, but in practice all contributions of these integrals to the polarizability can be rewritten so that they do not require explicit calculation of the all-virtual quantities, by reordering the summation over indices.

\section{Results and Conclusions}

Frequency-dependent polarizabilities are calculated for the $\mathrm{Li}$ and Ar atoms, and the $\mathrm{HCl}, \mathrm{CO}, \mathrm{N}_{2}, \mathrm{O}_{2}$, and $\mathrm{H}_{2} \mathrm{O}$ molecules, with the molecular geometries fixed at approximately their ground-state vibrationally averaged values. The bond lengths are $2.409 a_{0}$ for $\mathrm{HCl}, 2.132 a_{0}$ for $\mathrm{CO}, 2.081 a_{0}$ for $\mathrm{N}_{2}, 2.288 a_{0}$ for $\mathrm{O}_{2}$, and the origin of coordinates is taken to be the $\mathrm{Cl}$ atom for $\mathrm{HCl}$, and a point midway between the nuclei for the other three diatomics. For the water molecule, the oxygen atom is placed at the origin of coordinates, and the hydrogen atoms at $( \pm 1.4537,0,-1.12167) a_{0}$. The SIMPER program ${ }^{10,11}$ is used to perform the quantum chemical calculations, and the CCSD energies and densities are checked for consistency against other programs.

The methods used to calculate the frequency-dependent polarizabilities are the TD-CCSD method described in this article, as well as the TD-MP2 ${ }^{12}$ and TD-CHF ${ }^{13}$ methods, which are defined by replacing the CCSD Lagrangian in eq. (7) by the Møller-Plesset and Hartree-Fock Lagrangians respectively, and the TD-UCHF (timedependent uncoupled Hartree-Fock) method, which is defined using eq. (4), with the "excited states" defined as single excitations $i \rightarrow a$ from the Hartree-Fock ground state, and the energy differences defined as the difference of the two orbital energies $\epsilon_{a}-\epsilon_{i}$. All electrons are active in all the calculations, and the open-shell species $\mathrm{Li}$ and $\mathrm{O}_{2}$ are treated using unrestricted electron spins.

From the resulting frequency-dependent polarizabilities, dispersion energy coefficients are calculated for interactions between homomolecular pairs using eq. (3). This produces a large number of dispersion energy coefficients for each interaction. To reduce the amount of data presented, only the spherically averaged $C_{6}$ and $C_{8}$ dispersion energy coefficients, $C_{6}^{0}$ and $C_{8}^{0}$, are reported in this article. They are defined as

$$
C_{n}^{0}=\sum_{l}\left(\begin{array}{c}
2 l+2 l^{\prime} \\
2 l
\end{array}\right)\left\langle C_{\mathrm{disp} ; \lambda, \lambda, \lambda^{\prime}, \lambda^{\prime}}\right\rangle
$$

where $2 l+2 l^{\prime}+2=n$, and the average is over all $\kappa$ and $\kappa^{\prime}$. For the $C_{6}$ coefficient, this gives

$$
\begin{array}{r}
C_{6}^{0}=6\left(C_{\mathrm{disp} ; x, x, x, x}+C_{\mathrm{disp} ; x, x, y, y}+C_{\mathrm{disp} ; x, x, z, z}+C_{\mathrm{disp} ; y, y, x, x}\right. \\
+C_{\mathrm{disp} ; y, y, y, y}+C_{\mathrm{disp} ; y, y, z, z}+C_{\mathrm{disp} ; z, z, x, x}+C_{\mathrm{disp} ; z, z, y, y} \\
\left.+C_{\mathrm{disp} ; z, z, z, z,}\right) / 9 .
\end{array}
$$


Table 2. Spherically Averaged Dispersion Energy Coefficients and Polarizabilities (in Atomic Units), Calculated Using the TD-UCHF, TD-CHF, TD-MP2, and TD-CCSD Methods with the SPQZ Basis Set and with Unrestricted Electron Spins.

\begin{tabular}{|c|c|c|c|c|c|c|}
\hline Method & TD-UCHF & TD-CHF & TD-MP2 & TD-CCSD & NR-CCSD & Literature \\
\hline \multicolumn{7}{|l|}{$\mathrm{Li}$} \\
\hline$\alpha^{0}$ & 52 & 170 & 167 & 166 & & 164 (ref. 14) \\
\hline$C_{6}^{0}$ & 327 & 1,393 & 1,356 & 1,346 & & 1,387 (ref. 14 ) \\
\hline$C_{8}^{0}$ & 25,670 & 79,470 & 77,430 & 76,820 & & \\
\hline \multicolumn{7}{|l|}{$\mathrm{Ar}^{\circ}$} \\
\hline$\alpha^{0}$ & 10.0 & 10.7 & 11.2 & 11.1 & 11.2 & 11.1 (ref. 15) \\
\hline$C_{6}^{0}$ & 76.2 & 62.0 & 65.8 & 65.1 & $66.3-66.5$ & 64.3 (ref. 15) \\
\hline$C_{8}^{0}$ & 1,521 & 1,547 & 1,667 & 1,642 & $1,663-1,667$ & \\
\hline \multicolumn{7}{|l|}{$\mathrm{HCl}$} \\
\hline$\alpha^{0}$ & 15.7 & 16.7 & 17.5 & 17.2 & 17.5 & 17.4 (ref. 15) \\
\hline$C_{6}^{0}$ & 152.8 & 122.0 & 129.0 & 126.7 & $129.7-131.9$ & 130.4 (ref. 15) \\
\hline$C_{8}^{0}$ & 3,976 & 3,990 & 4,296 & 4,187 & $4,252-4,304$ & \\
\hline \multicolumn{7}{|l|}{$\mathrm{CO}$} \\
\hline$\alpha^{0}$ & 11.3 & 12.3 & 13.2 & 13.0 & 13.1 & 13.1 (ref. 16) \\
\hline$C_{6}^{0}$ & 90.9 & 74.7 & 81.3 & 79.1 & $80.9-82.7$ & 81.3 (ref. 16) \\
\hline$C_{8}^{0}$ & 2,864 & 2,898 & 3,221 & 3,090 & $3,128-3,178$ & \\
\hline \multicolumn{7}{|l|}{$\mathrm{N}_{2}{ }^{8}$} \\
\hline$\alpha^{0}$ & 11.6 & 11.6 & 11.6 & 11.7 & 11.7 & 11.7 (ref. 14) \\
\hline$C_{6}^{0}$ & 97.7 & 73.2 & 72.2 & 72.9 & $73.8-74.7$ & 73.3 (ref. 14) \\
\hline$C_{8}^{0}$ & 2,545 & 2,441 & 2,571 & 2,537 & $2,550-2,575$ & \\
\hline \multicolumn{7}{|l|}{$\mathrm{O}_{2}{ }^{8}$} \\
\hline$\alpha^{0}$ & 9.5 & 11.3 & 9.3 & 10.6 & & 10.6 (ref. 14) \\
\hline$C_{6}^{0}$ & 71.9 & 62.9 & 57.4 & 61.2 & & 61.6 (ref. 14) \\
\hline$C_{8}^{0}$ & 1,692 & 1,742 & 1,762 & 1,813 & & \\
\hline \multicolumn{7}{|l|}{$\mathrm{H}_{2} \mathrm{O}$} \\
\hline$\alpha^{0}$ & 7.28 & 8.72 & 9.94 & 9.57 & 9.82 & 9.64 (ref. 14) \\
\hline$C_{6}^{0}$ & 42.3 & 40.8 & 48.0 & 45.6 & $46.9-47.9$ & 45.3 (ref. 14) \\
\hline$C_{8}^{0}$ & 895 & 1,016 & 1,261 & 1,174 & $1,205-1,225$ & \\
\hline
\end{tabular}

NR-CCSD bounds were obtained using the Dalton program, see the text for details. Literature values are taken from constrained dipole oscillator strength data.

The integration in eq. (3) is performed using a sum over $N$ integration points, obtained by applying the extended trapezium rule to $\tan ^{-1}(\omega)$ :

$$
C_{\mathrm{disp} ; \lambda_{1}, \lambda_{2} ; \lambda_{3}, \lambda_{4}}=\sum_{k=1}^{N} W_{k} \alpha_{\mathrm{A}, \lambda_{1}, \lambda_{2}}\left(i \omega_{k}\right) \alpha_{\mathrm{B}, \lambda_{3}, \lambda_{4}}\left(i \omega_{k}\right)
$$

where $\omega_{k}=\tan ((2 k-1) \pi /(4 N))$, and the weight $W_{k}=(1+$ $\left.\omega_{k}^{2}\right) /(4 N)$ includes the factor of $1 /(2 \pi)$ in eq. (3). Results are calculated with $N=5$, and some checks are made with $N=15$ to ensure that the numerical integration does not introduce significant errors. For the Li atom, the imaginary-frequency-dependent polarizabilities decrease much more quickly, as $\omega$ increases from zero, than they do for the other species. The numerical integration to obtain the dispersion energy coefficients for $\mathrm{Li}-\mathrm{Li}$ is therefore based on $\tan ^{-1}(10 \omega)$, so $\omega_{k}=\tan ((2 k-1) \pi /(4 N)) / 10$ and $W_{k}=\left(1+100 \omega_{k}^{2}\right) /(40 N)$.

Table 1 compares spherically averaged dispersion energy coefficients obtained with the TD-CCSD method using different basis sets. The basis sets denoted DZ, TZ, and QZ are the aug-cc-pVDZ, augcc-pVTZ, and aug-cc-pVQZ basis sets, respectively, ${ }^{17,18}$ whereas the SPTZ and SPQZ basis sets are modified versions of these, ${ }^{4,19}$ which are designed to improve the convergence of the higher dispersion energy coefficients by reducing the exponents of the polarization functions. The literature values quoted are obtained from scaled dipole oscillator strength distributions. They are believed to be the most reliable benchmark results in the literature and are claimed to be accurate to about $1 \%$.

The values of $C_{6}^{0}$ appear to have converged reasonably well, to about $1 \%$, with increasing basis set size. The exception is Ar, for which further calculations with basis sets of quintuple-zeta size indicate that the SPQZ results may be too high by $1 \%$ or more; the reason for this is not clear. The $C_{8}^{0}$ coefficients calculated with the aug-cc-pVnZ basis sets are consistently smaller, and converge less quickly with basis set size, than those calculated with the SP basis sets. The SPTZ and SPQZ results for $C_{8}^{0}$ are in sufficiently close agreement that the latter can be taken as a reasonable estimate of the basis set limit, to within about $2 \%$, with the possible exception of Ar. When the calculations are repeated using $N=15$ values of $\omega$ in the calculation of the dispersion energy coefficients, the difference compared with $N=5$ is always small. For example, the largest difference in $C_{6}^{0}$ is less than 0.02 for $\mathrm{Ar}$ and 0.05 for $\mathrm{Li}$, and for $C_{8}^{0}$ the differences are less than 0.4 and 6 , respectively. This is negligible compared with the differences between the basis sets. 
In general, the agreement between the TD-CCSD results and the literature values of $C_{6}^{0}$ is good. For $\mathrm{N}_{2}, \mathrm{O}_{2}$, and $\mathrm{H}_{2} \mathrm{O}$, the difference is less than the estimated $1 \%$ uncertainties in the results. This is also true for Ar, if the SPQZ result (1.2\% too large) is anomalous, as suggested earlier. The largest basis sets for $\mathrm{Li}, \mathrm{HCl}$, and $\mathrm{CO}$ give results that are $3 \%$ too small. These discrepancies could arise from several factors, particularly the neglect of core polarization functions in the basis set, the use of rigid molecules, and the truncation of the coupled-cluster expansion at single and double excitations.

Table 2 compares the TD-CCSD dispersion energy coefficients with dispersion energy coefficients obtained using the more approximate, and computationally cheaper, TD-UCHF, TD-CHF, and TD-MP2 methods. The spherically averaged static polarizability $\alpha^{0}$ is also shown in the table. The SPQZ basis set is used for these calculations, as it is expected to give the most reliable results, particularly for $C_{8}^{0}$.

The results generally reflect the expectation that accuracy will increase in the sequence $\mathrm{UCHF}<\mathrm{CHF}<\mathrm{MP} 2<\mathrm{CCSD}$. Although the TD-MP2 method is closer to the literature value of $C_{6}^{0}$ than the TD-CCSD method for several of the interactions, it gives discrepancies of more than $7 \%$ for $\mathrm{O}_{2}$ and $5 \%$ for $\mathrm{H}_{2} \mathrm{O}$, compared with a maximum discrepancy of $3 \%$ for TD-CCSD. The TD-CHF method gives discrepancies of about $10 \%$ for several interactions, and the TD-UCHF method fails badly for Li, and substantially overestimates $C_{6}^{0}$ for most of the other interactions. It should be noted that dispersion energy coefficients obtained from the TDUCHF method correspond exactly to the long-range limit of the dispersion interaction energy in an MP2 supermolecule calculation, so the MP2 method is expected to be equally unreliable for calculating the binding energies of these dimers, particularly the weakly bonded (high-spin) dimers of $\mathrm{Li}, \mathrm{Ar}, \mathrm{N}_{2}, \mathrm{O}_{2}$, and $\mathrm{CO}$, where the dispersion energy dominates the total binding energy.

For the TD-CHF, TD-MP2, and TD-CCSD methods, the trends in the polarizabilities generally match those in the $C_{6}^{0}$ dispersion energy coefficients. Larger polarizabilities usually correspond to larger $C_{6}^{0}$ and $C_{8}^{0}$ values, although there are several exceptions, and the TD-UCHF method does not behave in the same way. The CCSD polarizabilities are usually closer to the literature values than the other methods, with the maximum discrepancies being about $1 \%$.

Published calculations of polarizabilities and dispersion energy coefficients are generally in agreement with the results in Table 2. For example, the TD-CHF values of $C_{6}^{0}$ and $C_{8}^{0}$ calculated by Thakkar et al. for $\mathrm{Ar}^{20}$ agree with the results in the table to within $1 \%$, while those of Rijks and Wormer for $\mathrm{N}_{2},{ }^{21} \mathrm{CO},{ }^{22}$ and $\mathrm{H}_{2} \mathrm{O}^{22}$ are 1-3\% lower, presumably because they used smaller basis sets. The MBPT calculations reported in the same papers by Rijks and Wormer are not directly comparable with the present results, but the MBPT calculations of Wormer and Hettema ${ }^{23}$ used a different method which gives agreement to within $1 \%$ with the TD-MP2 results in this article for the dispersion energy coefficients of $\mathrm{H}_{2} \mathrm{O}$. The TD-CCSD results presented here are consistent with the value of $C_{6}^{0}=64.9$ obtained by Jaszunski et al. ${ }^{24}$ for the argon dimer, but the $\operatorname{CCSD(3)}$ approximation recently used by Korona et al. ${ }^{25}$ gives $C_{6}^{0}$ values more than $2 \%$ higher than TDCCSD for the argon dimer and the water dimer. The MP2 and
CCSD polarizabilities calculated by Christiansen et al. for $\mathrm{CO}$ (for example, Table III of their paper ${ }^{26}$ ) agree with the current results to within 0.05 atomic units; their polarizabilities for $\mathrm{N}_{2}$ are more than 0.1 atomic units smaller, but this may be because they use a shorter $\mathrm{N}-\mathrm{N}$ bond length. Christiansen et al. also calculated the effect of vibration on the polarizabilities, and concluded that it is significant ( $>0.1$ atomic unit) for $\mathrm{CO}$, but not for $\mathrm{N}_{2}$. This may help to explain the discrepancy between the TD-CCSD and best literature values for the dispersion energy coefficient $C_{6}^{0}$ of the $\mathrm{CO}$ dimer.

In conclusion, the TD-CCSD method, combined with the SPQZ basis set, gives $C_{6}$ dispersion energy coefficients within $3 \%$ or less of the best "experimental" literature values, for a number of different atoms and molecules. The open-shell species $\mathrm{Li}$ and $\mathrm{O}_{2}$ have been treated successfully using the TD-CCSD method with unrestricted electron spins, although the method would presumably not be applicable to molecules with a significant multireference character in their ground-state wavefunctions. The SPnZ series of basis sets gives $C_{8}$ coefficients that converge to the basis set limit much more quickly than the corresponding aug-cc-pVnZ basis sets, which have the same number of basis functions. (Further calculations show that this is also true for higher $C_{n}$ coefficients.) This is because the SPnZ basis sets improve the response of the Hartree-Fock orbitals to perturbations of higher angular momentum $(l>1)$, whereas the aug-cc-pVnZ basis sets are optimized for intramolecular correlation. For the $C_{6}$ coefficients, there is little to choose between the two types of basis sets, because calculating these coefficients involves only $l=1$ multipole perturbations, and the Hartree-Fock response of both basis sets to $l=1$ perturbation is similar. Christiansen et al. also found that using doubly-augmented (and larger) basis sets had little effect on the dipole properties. ${ }^{27}$

The results also suggest that the use of "relaxed" Hartree-Fock orbitals for calculating dispersion energy coefficients using the TDCCSD method is reasonable. Relaxed orbitals give results which are formally correct to a higher order in the correlation operator than nonrelaxed orbitals, ${ }^{26}$ and the advantage of using relaxed orbitals for frequency-independent properties, including the polarizability of $\mathrm{CO}$, has been demonstrated by direct comparison between the two methods. ${ }^{28}$ Additional calculations of dispersion coefficients using the CCSD method with nonrelaxed orbitals have been performed using the Dalton program ${ }^{29}$; upper and lower bounds on the dispersion coefficients were obtained by fitting a [3,4] Padé approximant to the Cauchy moments ${ }^{30}$ and the results are shown in Table 2 as "NR-CCSD". In general, the dispersion energy coefficients obtained from nonrelaxed orbitals appear to be larger, but there is not clear evidence in support of either relaxed or nonrelaxed orbitals.

The use of relaxed orbitals for frequency-dependent properties does have the possible disadvantage that poles in the (real)frequency-dependent polarizability occur at the TD-CHF excitation energies, as well as at the coupled-cluster excitation energies. However, calculating dispersion energy coefficients involves imaginaryfrequency-dependent polarizabilities $\alpha(i \omega)$, which do not have poles, and in practice they decrease smoothly from $\alpha(0)$ to zero as $\omega$ increases. This makes the possible disadvantage of using relaxed orbitals less serious, although it may be significant for $\mathrm{Li}$, for which the lowest excitation energy is small. 


\section{Acknowledgments}

The author thanks the Leverhulme Trust for a Study Abroad Fellowship and the Royal Society for a JWT Jones Travelling Fellowship. Dalton calculations were performed by Dr. T. Lillestolen.

\section{References}

1. Dham, A. K.; Allnatt, A. R.; Koide, A.; Meath, W. J. Chem Phys 1995, $196,81$.

2. Tang, K. T.; Toennies, J. P. J Chem Phys 2003, 118, 4976.

3. Wei, H.; Le Roy, R. J.; Wheatley, R.; Meath, W. J. J Chem Phys 2005, 122, 084321.

4. Tulegenov, A. S.; Wheatley, R. J.; Hodges, M. P.; Harvey, A. H. J Chem Phys 2007, 126, 094305.

5. Stone, A. J. The Theory of Intermolecular Forces; Clarendon: Oxford, 2002.

6. Price, S. L.; Stone, A. J.; Alderton, M. Mol Phys 1984, 52, 987.

7. Christiansen, O.; Jørgensen, P.; Hättig, C. Int J Quantum Chem 1998, 68,1 .

8. Koch, H.; Jensen, H. J. A.; Jørgensen, P.; Helgaker, T.; Scuseria, G. E.; Schaefer, H. F. J Chem Phys 1990, 92, 4924.

9. Pedersen, T. B.; Koch, H. J Chem Phys 1998, 108, 5194.

10. Bichoutskaia, E. N.; Hodges, M. P.; Wheatley, R. J. J Comput Methods Sci Eng 2002, 2, 391.

11. Bichoutskaia, E. N.; Tulegenov, A. S.; Wheatley, R. J. Mol Phys 2004, $102,567$.
12. Hättig, C.; Heß, B. A. Chem Phys Lett 1995, 233, 359.

13. Langhoff, P. W.; Epstein, S. T.; Karplus, M. Rev Mod Phys 1972, 44, 602.

14. Margoliash, D. J.; Meath, W. J. J Chem Phys 1978, 68, 1426.

15. Kumar, A.; Meath, W. J. Mol Phys 1985, 54, 823.

16. Kumar, A.; Meath, W. J. Chem Phys 1994, 189, 467.

17. Dunning, T. H. J Chem Phys 1989, 90, 1007.

18. Woon, D. E.; Dunning, T. H. J Chem Phys 1993, 98, 1358.

19. Bichoutskaia, E. N.; Hodges, M. P.; Wheatley, R. J. J Comput Methods Sci Eng 2002, 2, 391.

20. Thakkar, A. J.; Hettema, H.; Wormer, P. E. S. J Chem Phys 1992 , 97, 3252.

21. Rijks, W.; Wormer, P. E. S. J Chem Phys 1988, 88, 5704.

22. Rijks, W.; Wormer, P. E. S. J Chem Phys 1989, 90, 6507.

23. Wormer, P. E. S.; Hettema, H. J Chem Phys 1992, 97, 5592.

24. Jaszunski, M.; Rizzo, A.; Jørgensen, P. Theor Chem Acc 2001, 106, 251.

25. Korona, T.; Przybytek, M.; Jeziorski, B. Mol Phys 2006, 104, 2303.

26. Christiansen, O.; Hättig, C.; Gauss, J. J Chem Phys 1998, 109, 4745.

27. Christiansen, O.; Halkier, A.; Koch, H.; Jørgensen, P.; Helgaker, T. J Chem Phys 1998, 108, 2801.

28. Kobayashi, R.; Koch, H.; Jørgensen, P.; Lee, T. J. Chem Phys Lett 1993, $211,94$.

29. Dalton, a molecular electronic structure program, Release 2.0 (2005), http://www.kjemi.uio.no/software/dalton/dalton.html.

30. Hättig, C.; Christiansen, O.; Jørgensen, P. J Chem Phys 1997, 107,10592 